\title{
Hierarchical Self-Assembly Mechanism of Ladder-Like Orientated A $\beta 40$ Single-Stranded Protofibrils into Multistranded Mature Fibrils
}

\author{
Mengting $\operatorname{Tian}^{1}$, Bei $\mathrm{Li}^{2} *$, Lei Shen ${ }^{1 *}$ \\ ${ }^{1}$ School of Chemistry, Chemical Engineering and Life Science, Wuhan University of Technology, \\ Wuhan 430070, China \\ ${ }^{2}$ Research Center for Materials Genome Engineering, School of Materials Science and Engineering, Wuhan \\ University of Technology, Wuhan 430070, China
}

\section{Experimental section}

Materials. Amyloid- $\beta$ peptide with the human sequence DAEFRHDSGYEVHHQKLVFFAEDVGSNKGAIIGLMVGGVV A $\beta 40$ was obtained from AnaSpec Inc. (USA). Phosphate buffered saline (PBS) solution (50 mM, pH 7.4) was freshly prepared for all experiments. A $\beta 40$ PBS solutions without pre-aggregations and fibrils were prepared by an ultrafiltration method as described previously. ${ }^{1}$ First, $\mathrm{A} \beta 40$ lyophilized powder was dissolved in 1,1,1,3,3,3-hexafluoropropan-2-ol (HFIP) at a concentration of $1 \mathrm{mg} / \mathrm{mL}$ overnight. After removing HFIP by a gentle stream of argon gas followed by vacuum, A $\beta 40$ were dissolved with PBS under vortex. Then, A $\beta 40$ solution was filtrated through a $20 \mathrm{~nm}$ nanopore filter (Whatman Anotop 10) under an elongation flow at the rate of $3 \mu \mathrm{L} / \mathrm{s}$. The non-uniform pore structure of the filter is the key to obtaining the real monomers. Finally, A $\beta 40$ solution was centrifuged at $30000 \mathrm{~g}$ for $30 \mathrm{~min}$ and the top two-thirds of the solution was used for fibril formation experiments. $\mathrm{A} \beta 40$ fibril 
formation experiments were carried out by incubating the rigorously disaggregated $\mathrm{A} \beta 40$ chains in PBS $(20 \mu \mathrm{M})$ at $37^{\circ} \mathrm{C}$ and $\mathrm{pH} 7.4$ for 12 hours. Then, about $20 \mu \mathrm{L}$ aliquots of the incubation solution were deposited onto freshly cleaned mica substrates. After $10 \mathrm{~min}$, the mica substrates were gently rinsed with Milli-Q water and allowed to dry in the air for further AFM characterization.

Atomic Force Microscopy Characterization. The morphologies of $\mathrm{A} \beta 40$ fibrils were imaged on an atomic force microscopy (AFM, FM-Nanoview 1000) in a tapping mode. A silicon tip on nitride level (Budget Sensors Inc.) with $48 \mathrm{~N} / \mathrm{m}$ spring constant and $190 \mathrm{kHz}$ resonance frequency were used. The fibril morphologies were tracked through FiberApp ${ }^{2}$ to extract the height and contour length information from the corresponding AFM images with a step length $\sim 1$ pixel between two subsequent points along the fibril contour.

Molecular Dynamics (MD) Simulation. The MD simulations were carried out by using the GROMACS software ${ }^{3}$. The A $\beta 40$ peptides were modeled with the GROMOS54a7 force field and the water molecules via the simple point-charge (SPC) model. Each system was placed in a periodic rectangular box and the minimum distance between the peptides and the box edge was chosen as $1.5 \mathrm{~nm}$ to improve computational efficiency. For the simulations, the system was initially energy minimized using the steepest decent method and then followed by a 2.5 ns run to relax the solvent with all heavy atoms of the peptides consecutively restrained with force constants of $1000,500,200,50$ and $5 \mathrm{~kJ} \cdot \mathrm{mol}^{-1} \cdot \mathrm{nm}^{-2}$. After that, the peptide system was further equilibrated for another $30 \mathrm{~ns}$ without position restraint using the isobaric isothermal ensemble at $300 \mathrm{~K}$ and 1.0 bar. The Berendsen scheme was adopted to control both temperature and pressure with the time constants of $0.2 \mathrm{ps}$ and $1.0 \mathrm{ps}$, respectively. The water molecules were constrained 
using the SETTLE algorithm ${ }^{4}$, while the covalent bonds within the peptides were constrained using the LINCS algorithm ${ }^{5}$. The van der Waals interactions were cut off at $12.0 \AA$. The same cutoff radius was also used for the electrostatic interactions, beyond which the particle mesh Ewald approach was employed. The Newton's equations of motion were integrated using a leap-frog algorithm with a time step of $2.0 \mathrm{fs}$. In order to analyze hydrogen bonds, a geometric criterion was used in which the formation of a hydrogen bond was defined by both atom distance and bond orientation..$^{6,7}$ The combination of donor $\mathrm{O}$, hydrogen $\mathrm{H}$, and accepter $\mathrm{O}$ with an $\mathrm{O}-\mathrm{H} \cdots \mathrm{O}$ configuration was considered as formed only when the hydrogen-acceptor distance was shorter than $3.0 \AA$ and the angle of the hydrogen-donor-acceptor triplet was smaller than $30^{\circ}$.
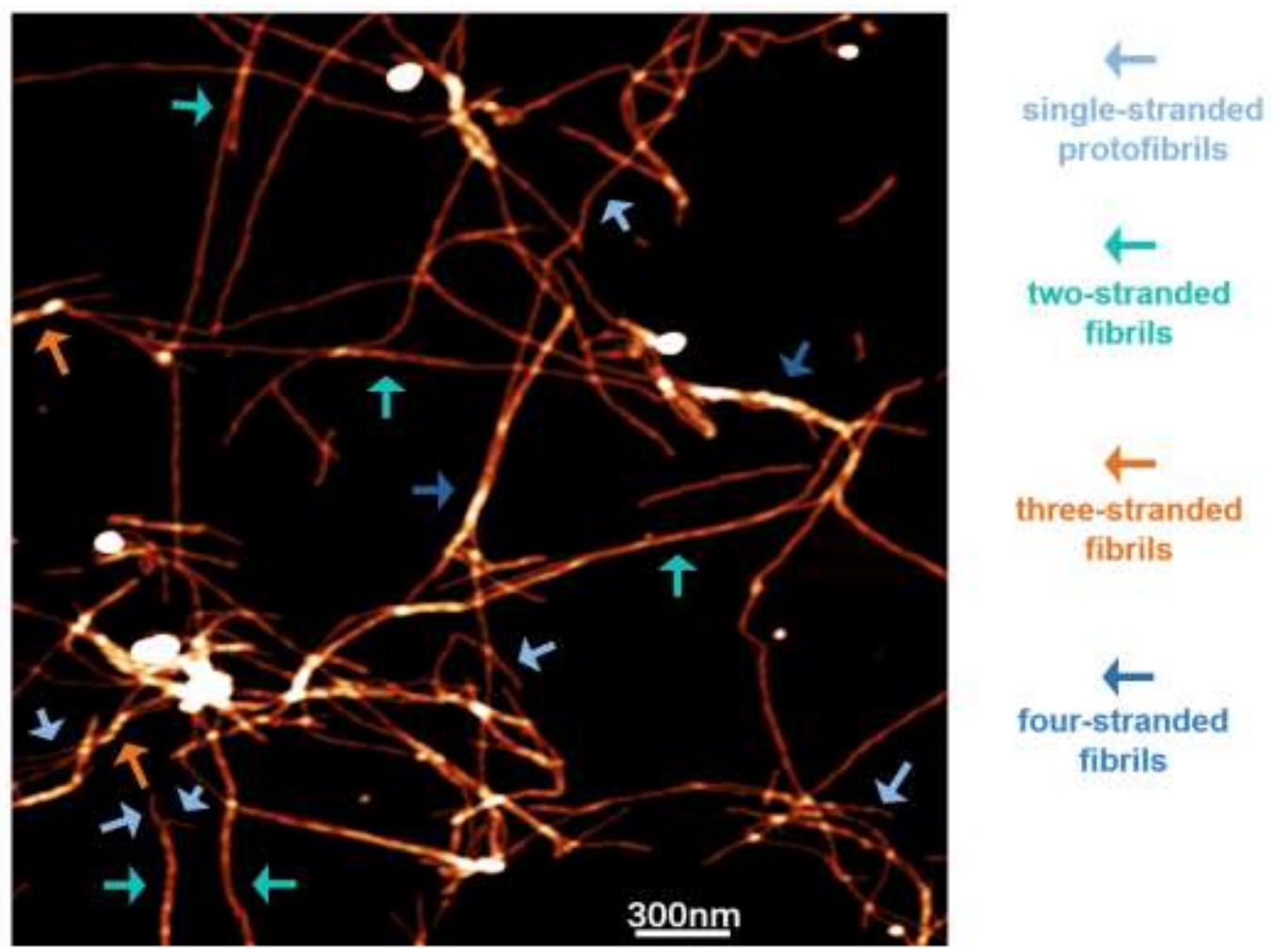

Figure S1. Example AFM height image of a larger view of the fibril structures formed via a quiescent incubation in PBS for $12 \mathrm{~h}$ at $37^{\circ} \mathrm{C}$ and $\mathrm{pH}$ 7.4. 


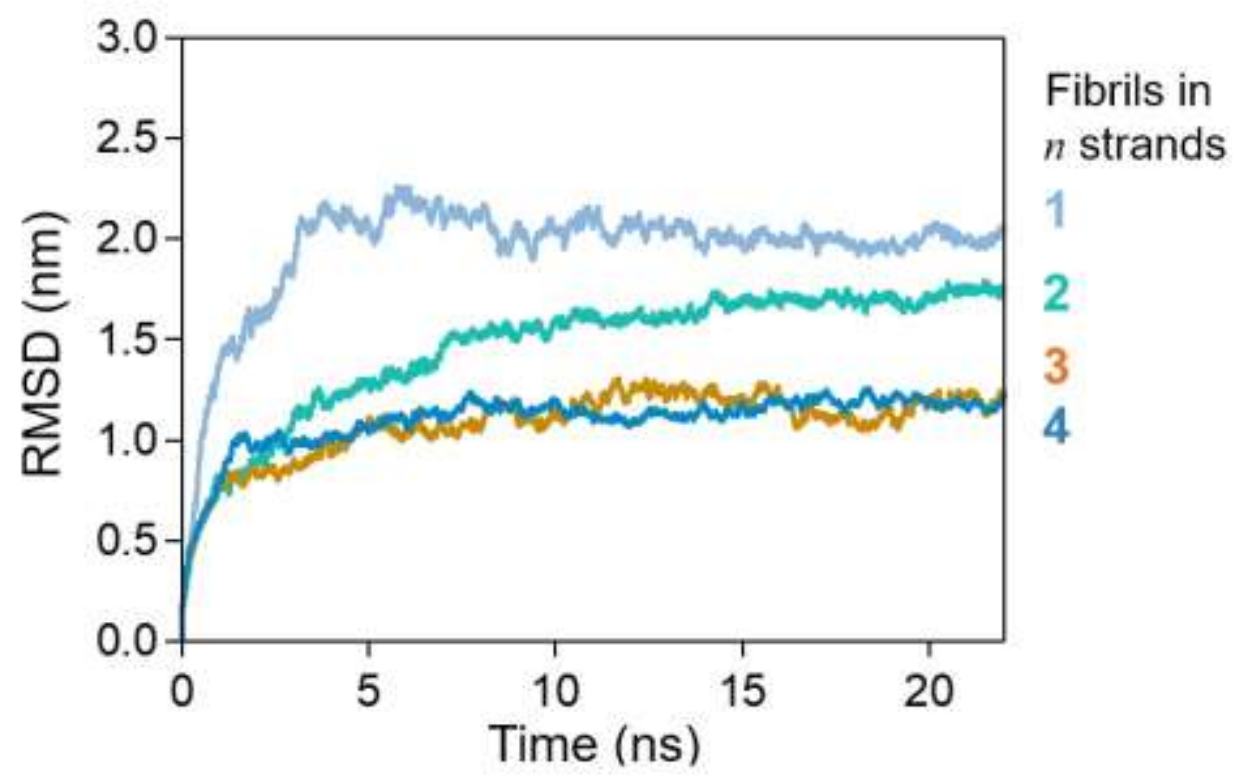

Figure S2. Time evolutions of the root mean square deviations (RMSDs) of $\mathrm{A} \beta 40$ fibrils in one, two, three, and four strands.
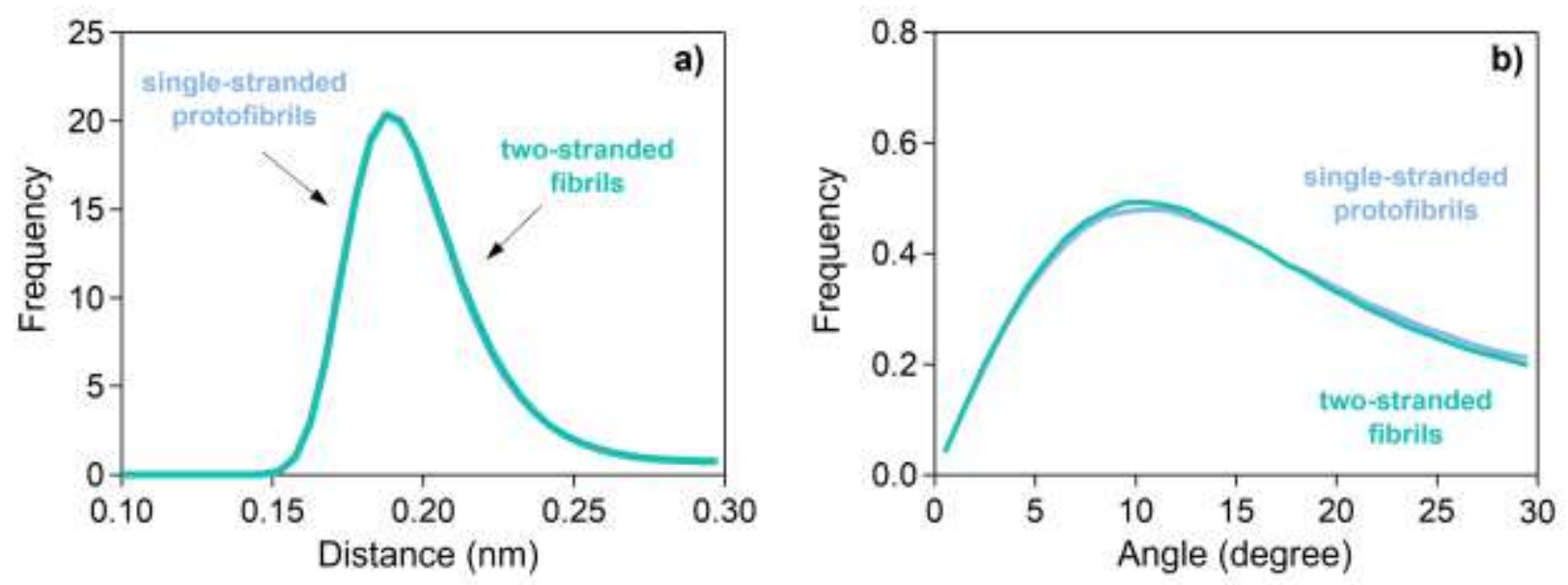

Figure S3. Distributions of hydrogen bond (a) distances and (b) angles for the A $\beta 40$ single-stranded protofibril and the $\mathrm{A} \beta 40$ two-stranded mature fibril.

1 Y. Wang, C. Wu, Biochemistry 2017, 56, 6575-6584.

2 I. Usov, R. Mezzenga, Macromolecules 2015, 48, 1269-1280.

3 B. Hess, C. Kutzner, D. van der Spoel, E. Lindahl, J. Chem. Theory Comput. 2008, 4, 435-447.

${ }^{4}$ S. Miyamoto, P. A. Kollman, J. Comput. Chem. 1992, 13, 952-962.

5 B. Hess, H. Bekker, H. J. C. Berendsen, J. G. E. M. Fraaije, J. Comput. Chem. 1997, 18, 1463-1472.

6 J. Russo, H. Tanaka, Nat. Commun. 2014, 5, 3556.

7 G. Chen, A. Li, H. Liu, S. Huang, Z. Zhang, W. Liu, C. Zha, B. Li, Z. Wang, Compos. Struct. 2018, 190, 160-168.

${ }^{8}$ B. Li, S. Hong, X. Zhang, C. Xiong, G. Zhao, Q. Yang, H. Liu, Adv. Theo. Simul. 2019, 2, 1900155. 